\title{
Problematic Use of Mobile Phones during the COVID-19 Pandemic in Peruvian University Students, 2020
}

\author{
Rosa Perez-Siguas ${ }^{1}$, Randall Seminario-Unzueta ${ }^{2}$ \\ Hernan Matta-Solis ${ }^{3}$, Melissa Yauri-Machaca ${ }^{4}$, Eduardo Matta-Solis ${ }^{5}$ \\ Research Directorate, Universidad María Auxiliadora, Lima, Perú ${ }^{1,3}$ \\ Research Unit, Universidad María Auxiliadora, Lima, Perú \\ Research and Technology Direction, Business on Making Technologies, Lima, Perú ${ }^{4}$ \\ Research Unit of the Faculty of Health Sciences, Universidad María Auxiliadora, Lima, Perú ${ }^{5}$
}

\begin{abstract}
The problematic use of mobile phones during the COVID-19 pandemic has been predicted as a mental alteration in university students due to confinement due to the health crisis that exists in our country and in the world, therefore, the objective of the study is to determine the problematic use of mobile phones during the COVID-19 pandemic. COVID-19 in Peruvian university students, 2020. This is a quantitative, nonexperimental, descriptive, and cross-sectional study, with a population of 163 Peruvian university students, who answered a questionnaire of sociodemographic data and the Mobile Phone Problem Use Scale. In the results where it can observe regarding the problematic use of mobile phones that $103(63.2 \%)$ of the university students have a high problematic use, $59(36.2 \%)$ medium problematic use and $1(\mathbf{0 . 6 \%})$ use low problematic use. In conclusion, programs on mental health should be carried out during the COVID-19 pandemic in university students.
\end{abstract} phones

Keywords-Mental health; pandemic; coronavirus; mobile

\section{INTRODUCTION}

Worldwide, mobile phones have become an important electronic device of the population as a means of communication [1], although excessive use has generated controversy and addictions within the young population, where they use mobile phones just for socialize by social networks and entertainment [2][3].

Although it is true, we are in a global health crisis due to the coronavirus pandemic (COVID - 19), today mobile phones are being used in a very excessive way, generating difficulties in their physical and mental health in the population [4], seeking factors such as anxiety, insomnia and stress, hindering the quality of life that had previously before the COVID -19 pandemic [5].

Likewise, the use of mobile phones has allowed the population to find a way to be entertained by the COVID-19 pandemic, but it also produces changes in the person's mood such as anger, sadness, depression, impulsivity [6], which are manifested as being isolated and quarantined as a result of the pandemic [7]. In such a way that it compromises the state of health of people, although it primarily affects the youngest because they are the ones who use mobile phones the most [8][9].

If the COVID-19 pandemic has caused young people to use their phones more, generating emotional and intellectual changes that compromise their academic performance affecting their daily lives, consequently young people will present long or short mental changes once the COVID-19 pandemic has subsided [10].

In China [11], a study was carried out in 754 adolescent students where $48.10 \%$ were women who had experience in the use of the mobile phone, where it was found that the time that adolescents spend on the mobile phone was related more to mobile addictions and psychological anguish, therefore, Chinese adolescents tended to have addiction in relation to the use of mobile phones for using them for a long time daily.

Similarly in China [12], a study was conducted in 847 university students, $51.2 \%$ men and $48.8 \%$ women, on the relationship between excessive use of mobile phones and alexithymia, where they observed that alexithymia predicts addiction to excessive use of mobile phones, whether directly or indirectly for entertainment.

In Spain [13], a study was carried out in 124 university students of the nursing career $79 \%$ were women and $21 \%$ were men, in relation to the use of mobile phones and nomophobia, observing that the male gender showed a lower tendency than the women in the use of mobile phones, concluding that the excessive use of mobile phones in university students are affected in their academic performance, as well as the relationship with patients and their colleagues at the time of carrying out their pre-professional practices.

Another study in Spain [14], argued that the excessive use of mobile phones taking the time of use, the age and the academic qualifications of the university students are factors that influence their self-esteem, therefore the misuse of mobile phones of students can generate addictive behaviors in the short and long term, compromising their health status and generating low self-esteem. 
In Malaysia [15], a study was conducted in 119 students where they stated in their results that those who did not use mobile phones had a better recovery in terms of learning and memory, while those who used mobile phones showed that it affects considerably learning and memory of students.

The objective of the research work is to determine the problematic use of mobile phones during the COVID-19 pandemic in Peruvian university students, 2020. This study is important since it will provide us with relevant and real data about the mental vulnerability and addiction that university students have during the pandemic.

\section{Methodology}

In this part, the type and design of the research will be developed, as well as the population and sample that will be carried out in the research work, the inclusion criteria in detail and finally the data collection technique and instrument.

\section{A. Research Type and Design}

The present study, due to its characteristics, way of collecting data and measuring the variables involved, has a quantitative approach. Regarding the methodological design, it is a non-experimental, descriptive, cross-sectional study [16].

\section{B. Population and Sample}

In this research work, it will be made up of 163 university students from Lima, Piura, and Trujillo.

\section{Inclusion Criteria}

- University students who are studying from the $1 \mathrm{st}$ semester to the 10th semester.

- University students who agree to be voluntarily participate in the research work.

\section{Technique and Instrument}

The technique used is the virtual Google form survey, in which the objective of the Mobile Phone Problem Use Scale (MPPUS) data collection instrument is to measure the problematic use of mobile phones during the COVID-19 pandemic in Peruvian university students, 2020.

For data collection, it was structured in two blocks: 1) Sociodemographic data such as age, sex, marital status, type of family, university, career and semester of study; 2) Problematic use of mobile phones (MPPUS) that contains 27 items valued on a Likert-type scale where " 1 = totally disagree", " $2=$ disagree", " $3=$ neither agree nor disagree", "4 $=$ agree" and " $5=$ totally agree", the final score ranges from 27 to 135 , where "27 to 50 is low problem use", "51 to 74 is medium problem use" and "75 to 135 high problem use", the higher the score corresponds to the more problematic use of mobile phones [17].

High problematic use refers to the excessive time or management that the university student has with the use of mobile phones, medium problematic use, refers to the time or habitual management that the university student has in the use of mobile phones and low problematic use, is refers to the time or occasional management that the university student has in the use of mobile phones during the COVID - 19 pandemic.

Regarding the validation and reliability results, they were obtained using the Kaiser-Meyer-Olkin sampling adequacy tests and the Bartlett sphericity test. The results of the sphericity test are significant while the sample adequacy test obtained a value of $0.538(\mathrm{KMO}>0,6)$ and the results of the sphericity test obtained a favorable value $\left(\mathrm{X}^{2}=1570,572\right.$; g.l. $=351 ; \mathrm{p}=0,000)$. Likewise, Cronbach's alpha index obtained a value of $0.684(\alpha>0,6)$. All the above tests confirm an acceptable index of validity and reliability of the instrument.

\section{E. Place and Application of the Instrument}

The virtual survey was carried out to measure the problematic use of mobile phones due to the COVID-19 pandemic in university students from different regions of Peru, in which it was carried out in Universities of Lima, Piura and Trujillo.

In order to start the data collection process, it coordinated with university students from the Universidad de Ciencias y Humanidades, Universidad César Vallejo, Universidad Privada del Norte, Universidad de Piura, Universidad Nacional de Cajamarca, Universidad Técnologica del Perú and Universidad San Martin de Porres, to voluntarily participate in research work, although there were certain limitations since not all students were willing to participate in research work, for work reasons or other corresponding factors in virtual classes or at home.

Likewise, the virtual survey of Peruvian university students is based on modern technology that they predispose for their management and in what way they spend time on it, therefore, the way in which university students can adapt during the COVID-19 pandemic to the use of modern technology in mobile phones.

\section{RESULTS}

Below is a summary table of the surveys carried out following the guidelines corresponding to the research work:

Fig. 1 shows the data of the university students in the study, where it can be observed with respect to the problematic use of mobile phones that $103(63.2 \%)$ of the university students have a high problematic use, $59(36.2 \%)$ medium problem use and $1(0.6 \%)$ low problem use.

This is since many of the university students tend to exceed the prudent time of using the technology of mobile phones exceeding between 8 to 12 corresponding hours, due to this each time the use of mobile phones becomes more problematic and that can generate an addiction in it.

Fig. 2 relates to problematic use of mobile phones and the sex of university students, where $77(66.4 \%)$ of the female sex have a high problematic use and 39 (33.6\%) of the female sex have a medium problem use, in males $26(63.2 \%)$ have high problem use, $20(36.2 \%)$ have medium problem use and 1 $(0.6 \%)$ have low problem use. 
Problematic Use of Mobile Phones

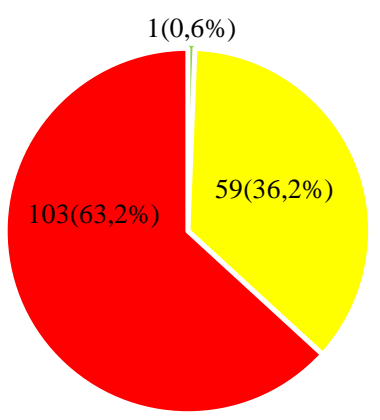

Low Problematic Use Medium Problematic Use

- High Problematic Use

Fig. 1. Problematic use of Mobile Phones during the COVID-19 Pandemic in Peruvian University Students, 2020.

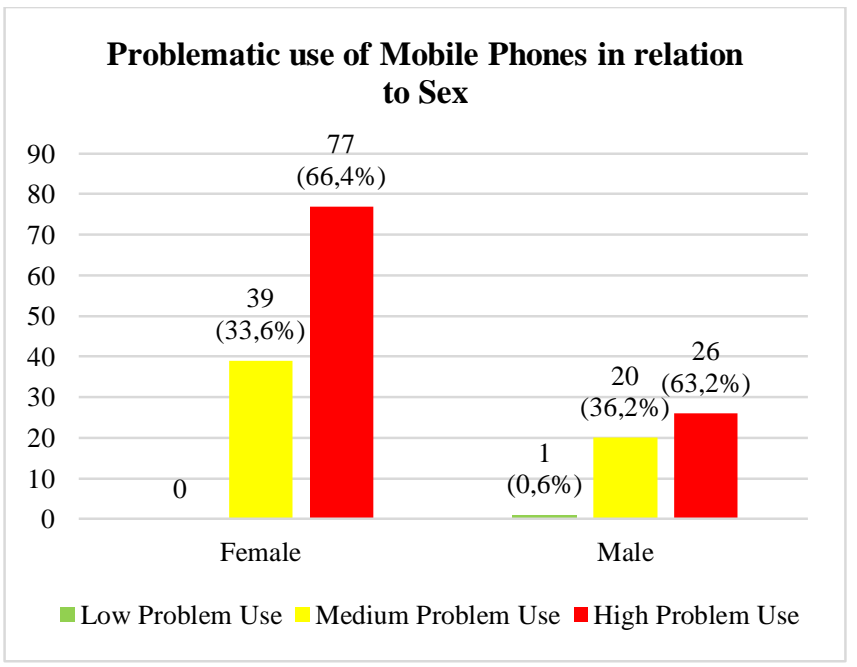

Fig. 2. Problematic use of Mobile Phones in relation to Sex during the COVID-19 Pandemic in Peruvian University Students, 2020
With respect to the female sex, a high index of problematic use of mobile phones was seen, this is because the female sex is more social than the male sex, because they are more aware of their emails or messages from friends that is why its use is considered extremely high.

Table I shows the problematic use of mobile telephones according to the university of Peruvian university students, in which it was determined with Pearson's chi-square test $\left(\mathrm{X}^{2}\right)$. The level of significance of the test obtained a value of 0.06 (p>0.05) $\left(\mathrm{X}^{2}=2,958\right.$; d.f $\left.=12\right)$. Therefore, an association hypothesis is not rejected, for which there are statistical data that verify the relationship between the problematic use of mobile phones and the University. By which, we can interpret that the university students of the Universidad de Ciencias y Humanidades have a high problematic use (62.5\%), as in the Universidad de Piura (58.3\%), the Universidad Privada del Norte $(58,1 \%)$, the Universidad Nacional de Cajamarca (60\%), the Universidad Técnologica del Perú (66.7\%), Universidad César Vallejo (71.4\%) and the Universidad San Martín de Porres (69.2\%).

In Table II, the problematic use of mobile phones is related according to the career of Peruvian university students, in which it was determined with Pearson's chi-square test $\left(\mathrm{X}^{2}\right)$. The level of significance of the test obtained a value of 0.06 $(p>0.05)\left(X^{2}=4,364\right.$; d.f $\left.=12\right)$. Therefore, an association hypothesis is not rejected, for which there are statistical data that verify the relationship between the problematic use of mobile phones and the career of university students. By which, it can interpret that the university students of the nursing career have a high problematic use (63.6\%), as well as the Economics career (58.3\%), the Administration career (60\%), the Law (66.7\%), Education (70\%) and Accounting (72\%), finally, university students in the Computer Systems Engineering career have a medium problematic use of mobile phones.

These results show us how university students handle the use of mobile phones during the COVID-19 pandemic, also will contribute to more future research work on the current research topic. 
TABLE I. Problematic USE OF MobILE PHONES IN RELATION TO THE UNIVERSITY DURING THE COVID-19 PANDEMIC IN PERUVIAN UNIVERSITY STUDENTS, 2020

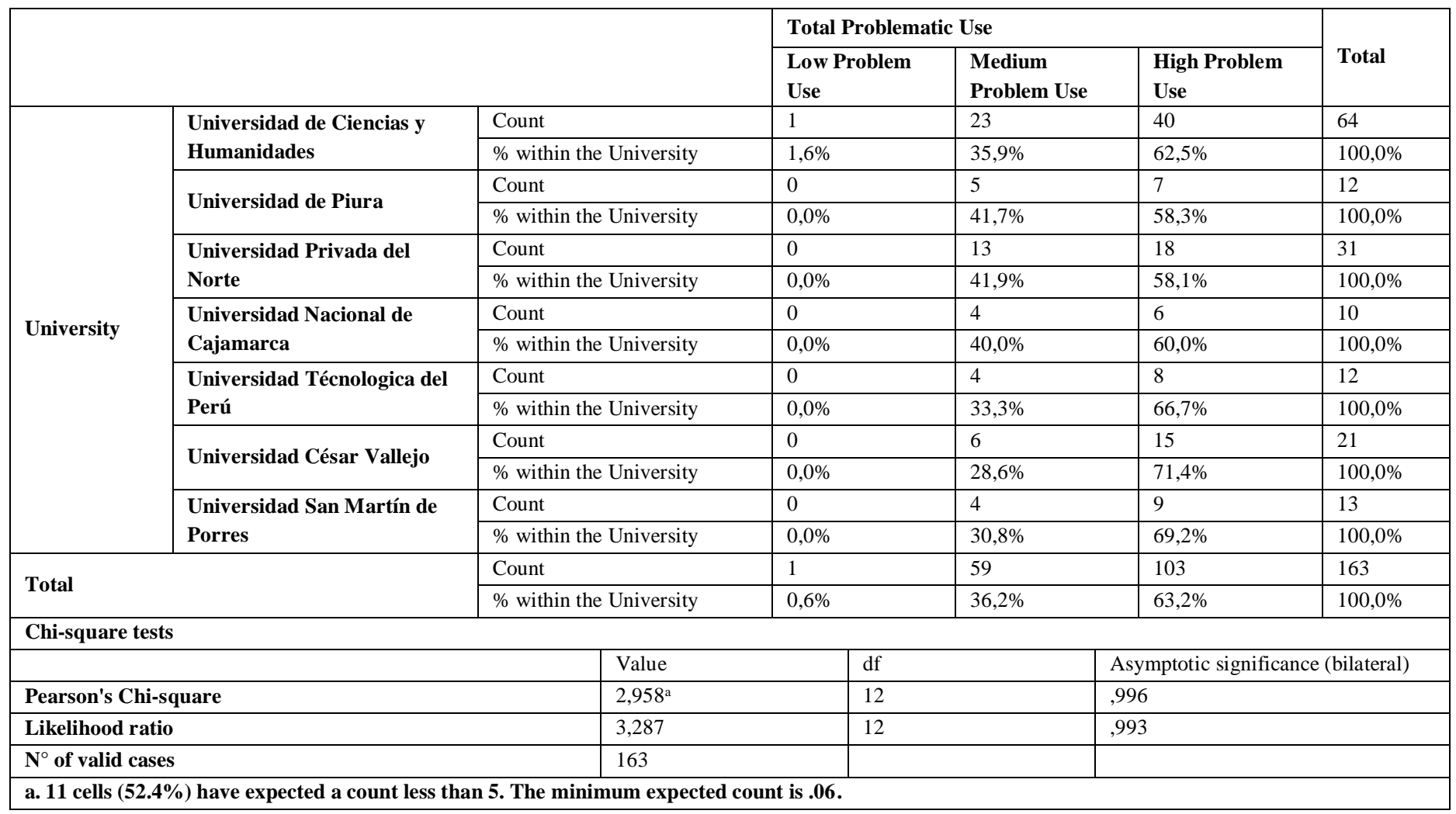

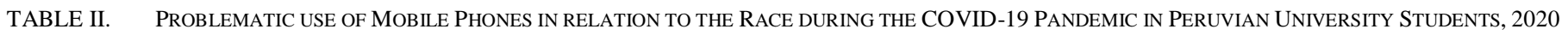

\begin{tabular}{|c|c|c|c|c|c|c|}
\hline & & & \multicolumn{3}{|l|}{ Total Overuse } & \multirow[b]{2}{*}{ Total } \\
\hline & & & $\begin{array}{l}\text { Low Problem } \\
\text { Use }\end{array}$ & $\begin{array}{l}\text { Medium } \\
\text { Problem Use }\end{array}$ & $\begin{array}{l}\text { High Problem } \\
\text { Use }\end{array}$ & \\
\hline & \multirow{2}{*}{ Nursing } & Count & 1 & 27 & 49 & 77 \\
\hline & & $\%$ within the University & $1,3 \%$ & $35,1 \%$ & $63,6 \%$ & $100,0 \%$ \\
\hline & \multirow{2}{*}{ Economy } & Count & 0 & 5 & 7 & 12 \\
\hline & & $\%$ within the University & $0,0 \%$ & $41,7 \%$ & $58,3 \%$ & $100,0 \%$ \\
\hline & \multirow{2}{*}{$\begin{array}{l}\text { Computer Systems } \\
\text { Engineering }\end{array}$} & Count & 0 & 9 & 8 & 17 \\
\hline & & $\%$ within the University & $0,0 \%$ & $52,9 \%$ & $47,1 \%$ & $100,0 \%$ \\
\hline \multirow{8}{*}{ Career } & \multirow{2}{*}{ Administration } & Count & 0 & 4 & 6 & 10 \\
\hline & & $\%$ within the University & $0,0 \%$ & $40,0 \%$ & $60,0 \%$ & $100,0 \%$ \\
\hline & \multirow{2}{*}{ Law } & Count & 0 & 4 & 8 & 12 \\
\hline & & $\%$ within the University & $0,0 \%$ & $33,3 \%$ & $66,7 \%$ & $100,0 \%$ \\
\hline & \multirow{2}{*}{ Education } & Count & 0 & 3 & 7 & 10 \\
\hline & & $\%$ within the University & $0,0 \%$ & $30,0 \%$ & $70,0 \%$ & $100,0 \%$ \\
\hline & \multirow{2}{*}{ Accounting } & Count & 0 & 7 & 18 & 25 \\
\hline & & $\%$ within the University & $0,0 \%$ & $28,0 \%$ & $72,0 \%$ & $100,0 \%$ \\
\hline To & & Count & 1 & 59 & 103 & 163 \\
\hline Total & & $\%$ within the University & $0,6 \%$ & $36,2 \%$ & $63,2 \%$ & $100,0 \%$ \\
\hline Chi-squ & ests & & & & & \\
\hline & & Value & c & & Asymptotic signifi & (bilateral) \\
\hline Pearson & i-square & $4,364^{\mathrm{a}}$ & & & ,976 & \\
\hline Likeliho & atio & 4,676 & & & ,968 & \\
\hline $\mathrm{N}^{\circ}$ of va & ases & 163 & & & & \\
\hline
\end{tabular}




\section{Discussion}

In this research work, a focus was provided on the mental health of university students in relation to the problematic use of mobile phones during their isolation due to the COVID-19 pandemic.

In the results on the problematic use of mobile phones, we can see that Peruvian university students present because of the COVID-19 pandemic, it has generated consequences in college students, such a degree that their psycho-emotional health has been affected, giving college students such as boredom, personality change, anxiety, and depression to such an extent that the feeling of having their mobile phone can help them manage their psycho-emotional state. In the study by $X$. Yang et al. [1], they argue that high levels of problematic mobile phone use are more related to boredom in college students, it generates undesirable psychosocial consequences in college students, such as anxiety and depression, impaired cognition, and poor academic performance.

In the results in relation to sex, we can observe that the female sex presented a high level of problematic use of mobile phones, this is because women are more attentive to their mobile phones since, they use it for their social networks, joint activities with friends, or are on the screen of their phones while talking by call with a person. In the study by J. Gao and collaborators [5], they argue that women are the ones who use social networks more on their mobile phones than men, because social networks are the only method to reduce their anxiety and depression, the COVID-19 pandemic is expected to affect the female population more than the male population.

In relation to the career, it was found that nursing students present a high level of problematic use of mobile phones, this is due to the fact that students, because of the COVID-19 pandemic, have had to stop their professional practices already also, take virtual courses either through their computers or mobile phones, generating stress and anxiety in themselves since they are not used to using technology on a daily basis since, as health students, they are more oriented to care and not to technology. In the study by V. Márquez et al. [13], they argue that female nursing students have a greater tendency to use mobile phones more than males, since females engage and maintain more active interpersonal relationships than males.

These aspects mainly encompass in mental health what $\mathrm{K}$. Chung et al. [6], maintain, referring that the inappropriate use of mobile phones compromises both the mental and physical health of the person, relating it to depressive symptoms, body pain and daytime sleepiness as predisposing factor. O. Király and collaborators [18], argue that the problematic use of mobile phones due to the COVID-19 pandemic has generated that the levels of uncertainty about the future and the insecurity, also generate a high level of stress, anxiety and depression because of this, therefore using mobile phones helps reduce those psycho-emotional factors that affect mental health due to confinement and quarantine.

\section{CONCLUSION}

It is concluded that programs on mental health should be carried out during the COVID-19 pandemic in university students.

It is concluded that the use of mobile phones should not be over-limited since it is compromised in the professional training of university students.

It is concluded that the use of technology for nursing students is detrimental in their academic performance, in decision-making, in the nurse-patient relationship and in the relationship with other colleagues from the career.

It is recommended that university students carry out a schedule of activities each day of the week, to allow them to carry out their daily routines at home during isolation or quarantine.

It is recommended to sleep regularly, have a healthy diet, take care of their personal hygiene, since they are essential to maintain good physical health and allows to improve their psychological well-being.

The limitation in this research work is that there are not many studies on the subject that are being carried out, both nationally and internationally.

\section{REFERENCES}

[1] X. Yang, Q. Liu, S. Lian, and Z. Zhou, “Are bored minds more likely to be addicted? The relationship between boredom proneness and problematic mobile phone use.," Addict. Behav., vol. 108, p. 106426, 2019, doi: 10.1016/j.addbeh.2020.106426.

[2] Z. Vally and F. El Hichami, "An examination of problematic mobile phone use in the United Arab Emirates: Prevalence, correlates, and predictors in a college-aged sample of young adults.," Addict. Behav. Reports, vol. 9, p. 100185, 2019, doi: 10.1016/j.abrep.2019.100185.

[3] G. Zhang, X. Yang, X. Tu, N. Ding, and J. Lau, "Prospective relationships between mobile phone dependence and mental health status among Chinese undergraduate students with college adjustment as a mediator.," J. Affect. Disord., vol. 260, pp. 498-505, 2020, doi: 10.1016/j.jad.2019.09.047.

[4] Hong W, Liu R, Ding Y, Sheng X, and Zhen R, "Mobile phone addiction and cognitive failures in daily life: The mediating roles of sleep duration and quality and the moderating role of trait selfregulation.," Addict. Behav., vol. 107, no. 19, p. 10638, 2020, doi: 10.1016/j.addbeh.2020.106383.

[5] J. Gao et al., "Mental health problems and social media exposure during COVID-19 outbreak.," PLoS One, vol. 15, no. 4, pp. 1-10, 2020, doi: 10.1371/journal.pone.0231924.

[6] K. Chun et al., "The relationships between mobile phone use and depressive symptoms, bodily pain, and daytime sleepiness in Hong Kong secondary school students.," Addict. Behav., vol. 101, p. 105975, 2020, doi: 10.1016/j.addbeh.2019.04.033.

[7] M. Romano, L. Osborne, R. Truzoli, and P. Reed, "Differential Psychological Impact of Internet Exposure on Internet Addicts.," PLoS One, vol. 8, no. 2, pp. 8-11, 2013, doi: 10.1371/journal.pone.0055162.

[8] H. Dong, F. Yang, X. Lu, and W. Hao, "Internet Addiction and Related Psychological Factors Among Children and Adolescents in China During the Coronavirus Disease 2019 (COVID-19) Epidemic," Front. Psychiatry, vol. 11, p. 751, 2020, doi: 10.3389/fpsyt.2020.00751.

[9] A. Ruiz, M. López, and J. López, "Evitación experiencial y uso abusivo del smartphone: un enfoque bayesiano," Adicciones, vol. 32, no. 2, p. 116, 2020, doi: 10.20882/adicciones.1151.

[10] S. Amez and S. Baert, "Smartphone use and academic performance: A literature review," Int. J. Educ. Res., vol. 103, p. 101618, 2020, doi: 10.1016/j.ijer.2020.101618. 
[11] S. Lian, X. Sun, G. Niu, X. Yang, Z. Zhou, and C. Yang, "Mobile phone addiction and psychological distress among Chinese adolescents: The mediating role of rumination and moderating role of the capacity to be alone.," J. Affect. Disord., 2020, doi: 10.1016/j.jad.2020.10.005.

[12] Z. Hao et al., "Alexithymia and mobile phone addiction in Chinese undergraduate students: The roles of mobile phone use patterns.," Comput. Human Behav., vol. 97, pp. 51-59, 2019, doi: 10.1016/j.chb.2019.03.001.

[13] V. Márquez, L. Puertas, G. Gámez, V. Puertas, and G. Manrique, "Problematic mobile phone use, nomophobia and decision-makin in nursing students mobile and decision-makin in nursing students.," Nurse Educ. Pract., vol. 49, p. 102910, 2020, doi: 10.1016/j.nepr.2020.102910.

[14] J. Romero and I. Aznar, “Análisis de la adicción al smartphone en estudiantes universitarios. Factores influyentes y correlación con la autoestima," Rev. Educ. a Distancia, vol. 60, no. 8, 2019, [Online]. Available: https://revistas.um.es/red/article/view/396051/273191.

[15] C. Tanil and M. Yong, "Mobile phones: The effect of its presence on learning and memory," PLoS One, vol. 15, no. 8, pp. 1-12, 2020, doi: 10.1371/journal.pone.0219233.

[16] C. Fernández and P. Baptista, Metodología de la Investigación. 6ta ed. México: Mc Graw-Hill/Interamericana. 2015.

[17] O. López, M. Honrubia, and M. Freixa, "Adaptación española del 'Mobile Phone Problem Use Scale' para población adolescente.," Adicciones, vol. 24, no. 2, p. 123, 2012, doi: 10.20882/adicciones.104.

[18] O. Király et al., "Preventing problematic internet use during the COVID-19 pandemic: Consensus guidance," Compr. Psychiatry, vol. 100, p. 152180, 2020, doi: 10.1016/j.comppsych.2020.152180. 